\title{
Enhanced tolerance to pulse extinction ratio in Brillouin optical time domain analysis sensors by dithering of the optical source
}

\author{
Haritz Iribas, Javier Urricelqui, Mikel Sagues and Alayn Loayssa \\ Universidad Pública de Navarra, Campus Arrosadia s/n, 31006 Pamplona, Spain.
}

\begin{abstract}
We demonstrate the relaxation of the stringent requirements placed on the pulse extinction ratio in long-range Brillouin optical time-domain analysis sensors (BOTDA) by modulating the wavelength of the laser source that is used to generate both pump and probe waves. This modulation makes the counter-propagating pulse pedestal and probe waves to become correlated only at certain locations in the fiber, thus reducing the gain experienced by the probe wave, which is precisely the process that limits the performance in long-range BOTDAs. Proof-of-concept experimental results in a $20-\mathrm{km}$ sensing link demonstrate a 6 - $\mathrm{dB}$ reduction of the required modulator extinction ratio.
\end{abstract}

Keywords: Brillouin distributed sensors, Brillouin optical time domain analysis, extinction ratio

\section{INTRODUCTION}

Brillouin optical time-domain analysis (BOTDA) sensors are a very interesting tool for the distributed measurement of temperature and strain over large structures, with very significant applications in civil and geotechnical engineering, in the monitoring of oil or gas pipelines or in assessment of high voltage cables. All these applications require a large sensing range. However, the sensing range of BOTDA sensors is limited by the maximum pump pulse power that can be injected in the fiber before the onset of nonlinear effects such as modulation instability or self-phase modulation(SPM). ${ }^{1}$ Moreover, it is also limited by the probe power that can be deployed, because, for large power, the energy exchanged between pump and probe via Brillouin interaction becomes significant so that the pump pulse becomes significantly depleted as it propagates along the fiber. This leads to so-called non-local effects, because the Brillouin spectra measured at distant locations of the fiber depends on the interaction at previous positions in the fiber. ${ }^{2}$

Another important factor limiting the performance for long-range BOTDAs is the pedestal or leakage of the pump pulses. ${ }^{2}$ Any practical device that is used to shape the pump pulses is bound to have a limited extinction ratio (ER). Therefore, in addition to the pulse, there is a continuous wave (CW) pump power that leaks and counter-propagates with the probe wave along the fiber, leading to an extra amplification of the latter. This extra amplification needs to be limited so as to avoid additional non-local effects and measurement errors, ${ }^{2,3}$ placing a very stringent requirement on the ER of the pump pulses ${ }^{2}$ used in long-range BOTDA. In this work, we demonstrate a novel method to alleviate these stringent specifications by taking advantage of the decorrelation of the pump pedestal and probe wave imposed by wavelength modulation of the optical source.

\section{FUNDAMENTALS OF THE TECHNIQUE}

The effect of the ER of the pump pulses on long-range BOTDA sensors is highlighted in Fig. 1a. These figure represents the various interactions that take place in the sensing fiber between the pump pulse with limited ER and the probe wave. The probe wave finds three regions of interaction with the pump wave as it travels along the fiber. The first one extends from the probe entrance in the fiber at $z=L$ to the location of the pulse at a particular $z_{0}$. In this region, just the pedestal of the pump pulse and the probe wave interact, leading to Brillouin amplification of the latter. Then, the probe wavefront meets the pulse and is amplified during the width $u$ of the pulse (half the spatial width of the pulse in the fiber, which equals the spatial resolution). Finally, after leaving

E-mail: alayn.loayssa@unavarra.es 


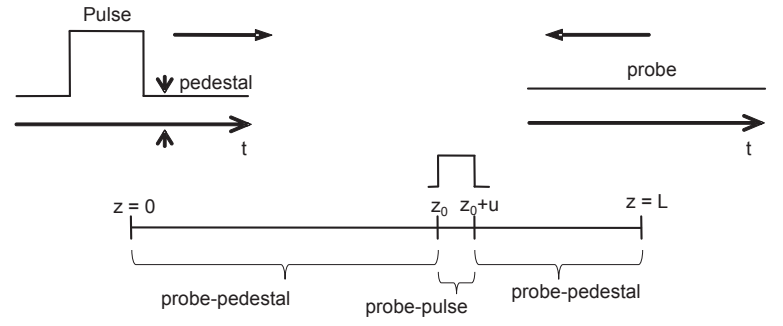

(a)

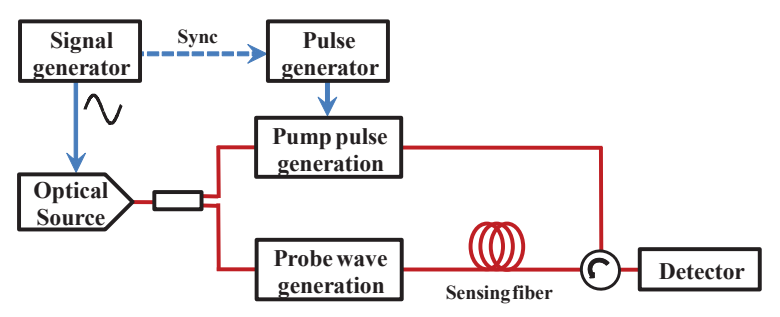

(b)

Figure 1: (a) Schematic depiction of probe and pulse waveform interaction along the fiber. (b) Principle of the laser wavelength dithering technique.

the pulse location, there is another region, from $z=z_{0}$ to $z=0$, where the probe wave is again amplified by the pulse pedestal. The net effect of the presence of the pulse pedestal is that the probe wave experiences more gain than just that due to the pulse itself. Besides, this is a gain that is not useful for sensing. On the contrary, it leads to detrimental effects because the measured gain spectrum due to interaction of the pulse and probe for a particular location $z=z_{0}$ in the fiber is the Brillouin gain spectrum for that location on top of another spectrum that is due to the pedestal with probe interaction integrated along the fiber. In principle, the Brillouin gain spectrum for $z=z_{0}$ can be obtained by normalizing the measurements by the spectrum measured with no pulse in the fiber. However, the additional gain in the probe wave has been shown to degrade the precision of the Brillouin frequency shift measurement. ${ }^{2,3}$ Moreover, the presence of the additional gain, apart from the useful signal, is limiting the amplitude of the probe wave that can be detected and processed in detection, leading to an effective reduction of the signal-to-noise ratio.

Limits to this detrimental effects have been defined by considering that, in order to have a negligible impact, the additional gain in the probe wave due to the pedestal needs to be smaller to the gain provided by the pulse itself. ${ }^{2}$ This translates to the condition that the lower bound for the extintion ratio must be $E R>L_{e f f} / u$, where $L_{e f f}$ is the effective fiber length that tends asymptotically to $L_{e f f} \approx 22 \mathrm{~km}$ for long-length fibers. Therefore the required ER scales with the spatial resolution. For instance, for 1 meter spatial resolution an ER larger than $43 \mathrm{~dB}$ is necessary. This is a very stringent requirement for the modulator or optical switch shaping the pulses. For instance, the typical ER of electrooptic modulators (EOM) is around 20 to $30 \mathrm{~dB}$. Specialty EOMs reach up to $40 \mathrm{~dB}$, but at much greater costs. On the other hand, cost-effective optical switches based on semiconductor optical amplifiers (SOA) are available with very large ER. However, SOAs are limited in the rise time of the pulses that they can produce, which is typically of the order of $1 \mathrm{~ns}$. This limits their ability to generate pulses that are immune to SPM degradation ${ }^{1}$ or to generate pulses for high spatial resolution applications using, for instance, the differential pulse-with pair technique. ${ }^{4}$

Therefore, there is a clear need to relax the requirements on ER of the devices used for generating the pump pulses. We propose to achieve this by dithering of the optical source used to generate the pump and probe waves. The principle is depicted in Fig. 1b. We directly modulate the injection current of a laser using a sinusoidal wave signal generator, which is synchronized to the pump pulse generator. This induces a modulation of the output optical signal wavelength via laser's chirp. We have previously shown that with this method we are able to generate a sort of virtual Brillouin frequency shift (BFS) profile along the fiber that, as it will be shown in the experimental results, mimics the shape of the chirp modulation and increases the tolerance of the sensor to non-local effects and spontaneous Brillouin scattering noise. ${ }^{5}$ However, when applied to a BOTDA sensor with limited-ER pulses, the effect is to reduce the Brillouin gain that the pulse pedestal provides to the probe wave. The mechanism is similar in principle to the interaction between pump and probe waves in Brillouin optical correlation domain analysis sensors. ${ }^{6}$ The pulse's pedestal and the probe wave have their wavelengths modulated with the same sinusoidal shape. When this two waves counter-propagate in the fiber, their wavelength difference is correlated just at certain locations in the fiber (correlation peaks). This correlation peaks are spaced by a distance $\nu_{g} / 2 f_{m}$, where $\nu_{g}$ is the group velocity of light in the fiber and $f_{m}$ is the wavelength modulation frequency. At all other locations in the fiber the pedestal and the probe become uncorrelated, thus reducing the energy transfer between both waves via Brillouin gain. Therefore, the parasitic gain given to the probe wave and 


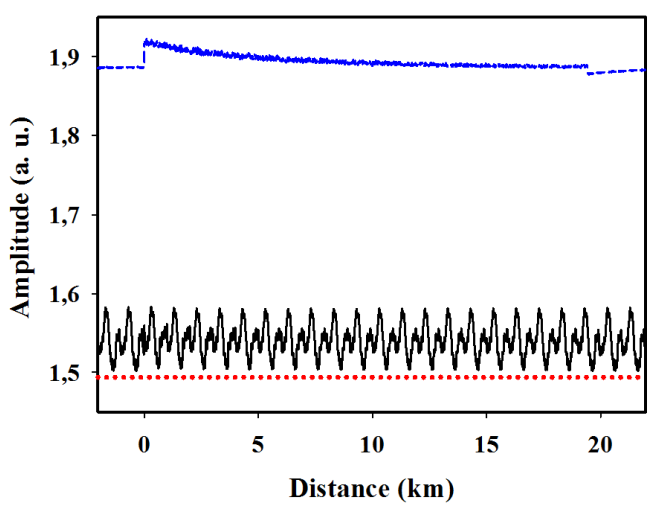

Figure 2: BOTDA signal measured for the conventional setup (blue dashed), for the setup with dithering of the optical source (black solid) and, finally, received probe wave without pump wave (red dotted).

causing the detrimental effects described above is greatly reduced, as it will be highlighted in next section.

\section{EXPERIMENTAL RESULTS AND DISCUSSION}

We assembled a BOTDA setup following the scheme in Fig. 1b. The optical source was a DFB laser at $1550 \mathrm{~nm}$ whose injection current was modulated by a $100-\mathrm{kHz}$ sinusoid from a signal generator. Pulses were generated in an electrical pulse generator that drove a Mach-Zehnder electrooptic modulator (MZ-EOM) with and ER of $28 \mathrm{~dB} \mathrm{~dB}$. This pulses where then amplified in an erbium doped amplifier (EDFA) before launching them into the sensing fiber. In the lower branch of the setup, another MZ-EOM is driven by a microwave generator with frequencies close to the BFS in the fiber. This modulator is biased for minimum transmission so as to generate a double-sideband suppressed-carrier modulation. This is a conventional BOTDA setup with two balanced probe waves and the only addition of the chirp modulation of the optical source. Before the detector, an optical filter is used to retain just the highest wavelength probe wave. Furthermore, a polarization switch is added after the MZ-EOM generating the probe wave, to smooth polarization effects in the Brillouin interaction along the fiber. Finally, in this proof-of-concept experiments a 20-km standard singlemode (ITU G.652) fiber spool with a fairly uniform BFS distribution was deployed as sensing fiber.

Fig. 2 depicts the improvement in ER effects brought by our technique by comparing measured BOTDA traces, i.e. probe wave signal as a function of time or distance, with and without optical source dithering. The detected probe wave without pump wave in the fiber is also shown. The trace for the conventional BOTDA is flat before the pulse enters the fiber, then we have the probe gain at every position due to interaction with the pulse and finally, there is another flat response. In the flat areas, before and after the pulse is in the fiber, we are just measuring the gain of the probe due to the Brillouin interaction with the pulse pedestal. Note that this gain is much larger than the gain due to the pulse interaction, which, as it was explained before, leads to errors in the measurements. Finally, we have the trace once the optical source dithering is turned on. Notice, that the gain in the areas without pulse in the fiber is greatly reduced. Also note that the gain in this areas is no longer flat, but displays an oscillatory behavior. The reason is that we are seeing the gain experienced by the probe wave as it meets wavefronts of the pulse's pedestal with varying wavelength difference. As a consequence, the Brillouin spectra are revealed once the gain due to the pulse pedestal is subtracted from the traces.

The relaxation of ER requirements brought by the application of our technique can be quantified by the reduction in probe gain, $g_{\text {probe }}$, due to the Brillouin interaction with the pedestal, which is given by:

$$
g_{\text {probe }}=\exp \left(\frac{g_{b}}{A_{e f f}} P_{\text {ped }} L_{e f f}\right)
$$

where $g_{b}$ is the Brillouin gain coefficient, $A_{e f f}$ the fiber's effective area and $P_{p e d}$ is the pulse's pedestal power. Using (1) we can find the equivalent enhancement in ER that would be associated to the reduction in probe gain 


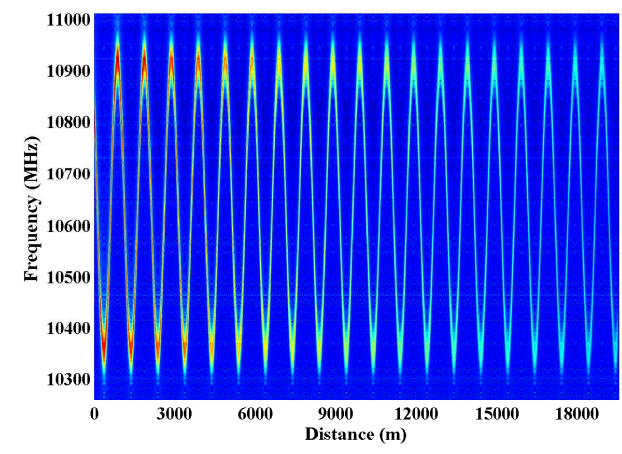

(a)

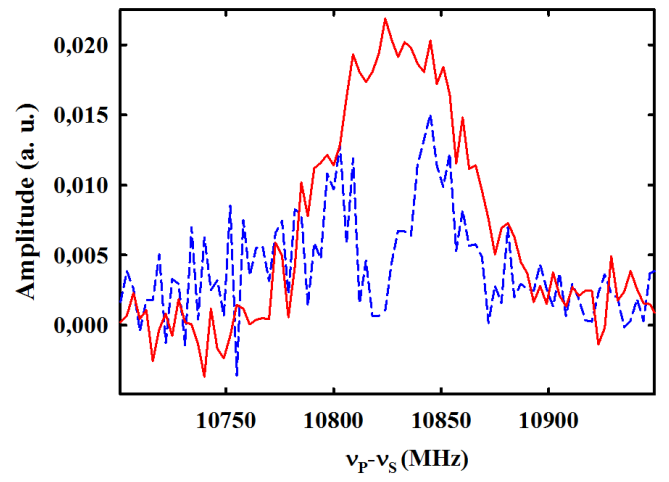

(b)

Figure 3: (a) Measured BFS distribution along the fiber. (b) Spectra at the output of the fiber with optical source dithering on (red solid) and off (blue dashed).

that we obtain with the optical source dithering technique. In this particular experiment the probe gain was reduced by a factor equivalent to a 6 - $\mathrm{dB}$ enhancement in the ER of the MZ-EOM.

Fig. 3a display the distribution of Brillouin spectra in the fiber for the BOTDA with optical source dithering. Note, that, as we have previously shown, ${ }^{5}$ the modulation of the wavelength of the optical source generates a virtual BFS profile that mimics the shape of this modulation, in this case a sinusoidal. The measured spectra at the output is shown in Fig. 3b and compared to that measured in a conventional BOTDA. Notice that in the latter case, the spectra is highly distorted at the output of the fiber. This can be attributed to the increased non-local effects induced by the amplification of the probe by a large pulse pedestal.

\section{CONCLUSIONS}

In summary, we have demonstrated the feasibility of the use of optical source dithering to reduce the pulse ER requirements in BOTDA sensors.

\section{ACKNOWLEDGMENTS}

We acknowledge the support of the Spanish Ministerio de Economía y Competitividad project TEC2013-47264C2-2-R, FEDER funds and the Universidad Pública de Navarra.

\section{REFERENCES}

[1] Foaleng, S., Rodrguez-Barrios, F., Martin-Lopez, S., Gonzlez-Herrez, M., and Thvenaz, L., "Detrimental effect of self-phase modulation on the performance of brillouin distributed fiber sensors," Optics Letters 36(2), 97-99 (2011).

[2] Thévenaz, L., Mafang, S., and Lin, J., "Effect of pulse depletion in a brillouin optical time-domain analysis system," Optics Express 21(12), 14017-14035 (2013).

[3] Zornoza, A., Olier, D., Sagues, M., and Loayssa, A., "Brillouin distributed sensor using rf shaping of pump pulses," Measurement Science and Technology 21(9), 094021 (2010).

[4] Li, W., Bao, X., Li, Y., and Chen, L., "Differential pulse-width pair botda for high spatial resolution sensing," Opt. Express 16, 21616-21625 (Dec 2008).

[5] Urricelqui, J., Sagues, M., and Loayssa, A., "Synthesis of brillouin frequency shift profiles to compensate non-local effects and brillouin induced noise in botda sensors," Optics Express 22(15), 18195-18202 (2014).

[6] Hotate, K., "Measurement of brillouin gain spectrum distribution along an optical fiber using a correlationbased technique-proposal, experiment and simulation-," IEICE Transactions on Electronics E83-C(3), 405$411(2000)$. 
Copyright 2015 Society of Photo Optical Instrumentation Engineers. One print or electronic copy may be made for personal use only. Systematic electronic or print reproduction and distribution, duplication of any material in this paper for a fee or for commercial purposes, or modification of the content of the paper are prohibited. 